\title{
Objective Assessment of Skin Tightening Using Multisource, Phase-Controlled Radiofrequency in Asians*
}

\author{
Yohei Tanaka ${ }^{1,2,3}$ \\ ${ }^{1}$ Clinica Tanaka Plastic, Reconstructive Surgery and Anti-Aging Center, Matsumoto, Japan; ${ }^{2}$ Department of Dermatology, Tokyo \\ Women's Medical University, Tokyo, Japan; ${ }^{3}$ Department of Applied Life Sciences, Niigata University of Pharmacy and Applied \\ Life Sciences, Niigata, Japan. \\ Email: info@clinicatanaka.jp
}

Received December $18^{\text {th }}, 2012$; revised January $20^{\text {th }}, 2013$; accepted January $28^{\text {th }}, 2013$

\begin{abstract}
Background: Radiofrequency has been proven to penetrate deeper than optical light sources independent of skin color allowing a safer treatment for the Asian skin type. Many studies have indicated the efficacy of various types of devices, but have not included a sufficient objective evaluation. Multisource radiofrequency uses multiple phase controlled radiofrequency generators with real time impedance control, allowing painless, deeper dermal heating with better adaptation to differences in individual skin impedance. In this study we used three-dimensional imaging for the objective evaluation of facial skin tightening by multisource phase-controlled radiofrequency. Methods: Twenty Japanese patients were treated with a multisource phase-controlled radiofrequency device. Three-dimensional imaging was performed with a Canfield Scientific Vectra camera and software, and quantitative volume measurements were taken to evaluate the change in the post-treatment volume. The patients then provided subjective assessments. Results: Objective assessments of the treated cheek volume evaluated by a three-dimensional color schematic representation with quantitative volume measurements showed significant improvement three months after the final treatment. The mean volume reduction at the last post-treatment visit was $3.878 \pm 2.86 \mathrm{~mL}$. The post-treatment volume was significantly reduced compared to the pretreatment volume in all of the volunteers $(P=0.0007)$. Ninety-five percent of volunteers reported satisfaction with the improvement of skin laxity, and ninety percent of volunteers reported satisfaction with the improvement of wrinkles, such as the nasolabial folds. Conclusions: The advantages of these multisource phase-controlled radiofrequency treatments are its high efficacy for skin tightening associated with minimal level of discomfort, minimal side effects, and low cost. Taken together, these characteristics facilitate the ability to give repeated treatments as a stand alone treatment or adjunct to surgery. This study provides for the first time a qualitative and quantitative volumetric assessment, proving the ability of the technology to reduce the volume through non invasive skin tightening.
\end{abstract}

Keywords: Objective Evaluation; Quantitative Volume Measurement; Skin Laxity; Three-Dimensional Imaging; Wrinkles

\section{Introduction}

Demand for a non-invasive and long-lasting treatment to reduce wrinkles and laxity has grown dramatically over the past few decades as new aesthetic technologies have been introduced into practice. A major cause of wrinkles, laxity and cellulite is the reduction in the quantity and quality of collagen in the dermis and hypodermis [1].

We previously reported that near-infrared can penetrate deep into human tissue, achieve skin tightening [24] and muscle thinning [5,6], and non-thermally induce

\footnotetext{
*Financial disclosure statement: I did the work without receiving any financial support from any third party. I have no relevant financial activities outside the submitted work.

Disclosure: None of the authors of this study have a conflict of interest.
}

various responses in the skin and subcutaneous tissues [7-12].

Radiofrequency (RF) devices have also been widely used for skin tightening and are thought to heat the dermis and subcutaneous tissues, thereby stimulating dermal collagen remodeling. It is well documented that one of the effects of dermal heating is an immediate change in collagen structure, followed by a long-term stimulation of neocollagenesis starting at 4-6 weeks after treatment [13]. These thermal effects can help reduce the appearance of wrinkles and laxity and improve contours on both the face and body.

The thermal effects of monopolar and bipolar RF have been proven to be beneficial in skin tightening. Never- 
theless, these effects are often partial or unpredictable because of the uncontrolled nature and possible pain produced during monopolar or unipolar RF treatments and the superficial nature of energy flow for bipolar or tripolar configurations. In addition these first-generation RF therapy systems delivered frequently unpredictable results, possibly due to the large differences in individual skin impedance [14].

In our clinic, a novel multisource phase-controlled system is used. In Multisource RF systems, first introduced in 2009, six independent RF generators produce the RF. Each of this generators is phase controlled, allowing a complex 3 dimensional interaction between the electromagnetic fields produced in the tissue. Since adjacent electrodes, on each side of the handpiece, posses identical polarities, no current is created between these electrodes on the skin's surface and most of the energy is driven deeper into the skin with minimal epidermal flow (Figures 1 and 2).

In addition to the new RF delivery technology, the tested system allows for continuous real-time measurement of skin impedance and delivers constant energy to the patient skin independent of changes in its impedance. Several studies have shown the efficacy of multisource RF in both skin tightening and fractional RF skin resurfacing but these studies did not include an objective evaluation of the post treatment volumetric tissue changes [1,14-17]. Conventional evaluations using photographs have been widely used, but they do not provide an accurate objective assessment. Therefore, in this study, a 3-dimensional (3D) photographic system was used to evaluate the amount of post-treatment volume change.

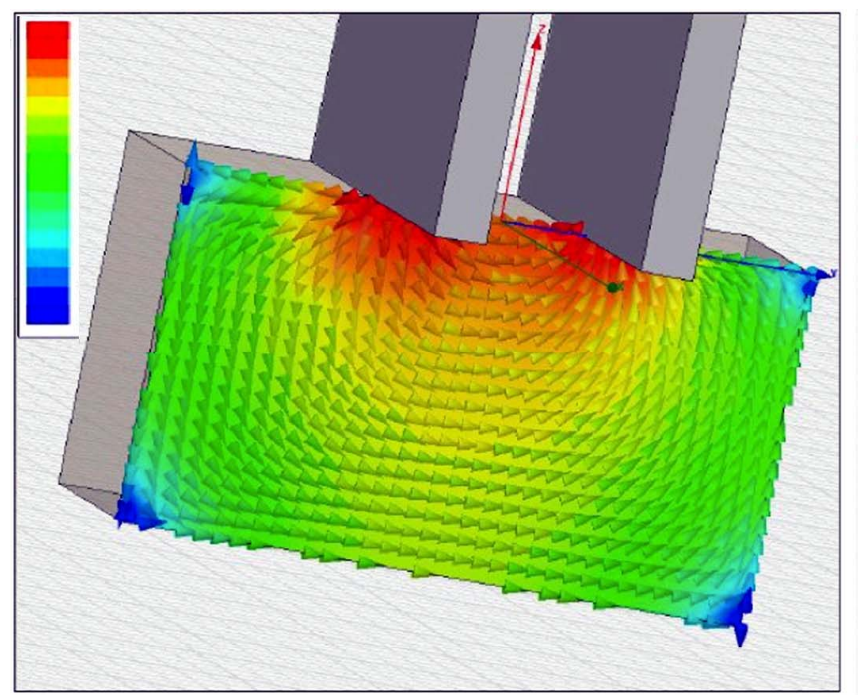

The aim was to quantifiably assess and provide definitive clinical evidence of skin tightening using multisource phase-controlled RF treatment not only subjectively, but also objectively.

\section{Materials and Methods}

\subsection{Japanese Volunteers}

Twenty Japanese volunteers (18 females and 2 males) aged 26 to 69 years (mean age, $42.4 \pm 9.92$ years) with

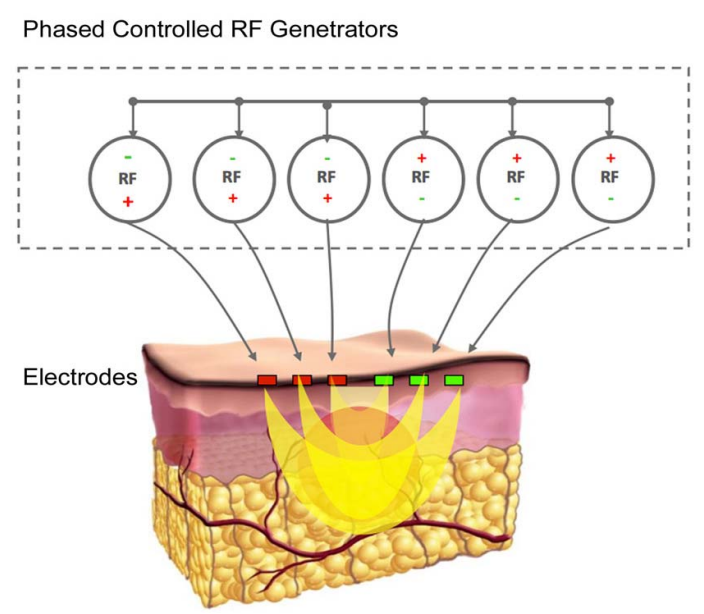

Figure 1. Configuration of six phase controlled RF sources as implemented in the Endymed Pro multisource technology. Multisource RF technology is based on the fact that flow of energy on the surface is minimal, while all energy is directed to the depth of the tissue. This is achieved by repulsion between electrical field of the same polarity on each side of the handpiece.

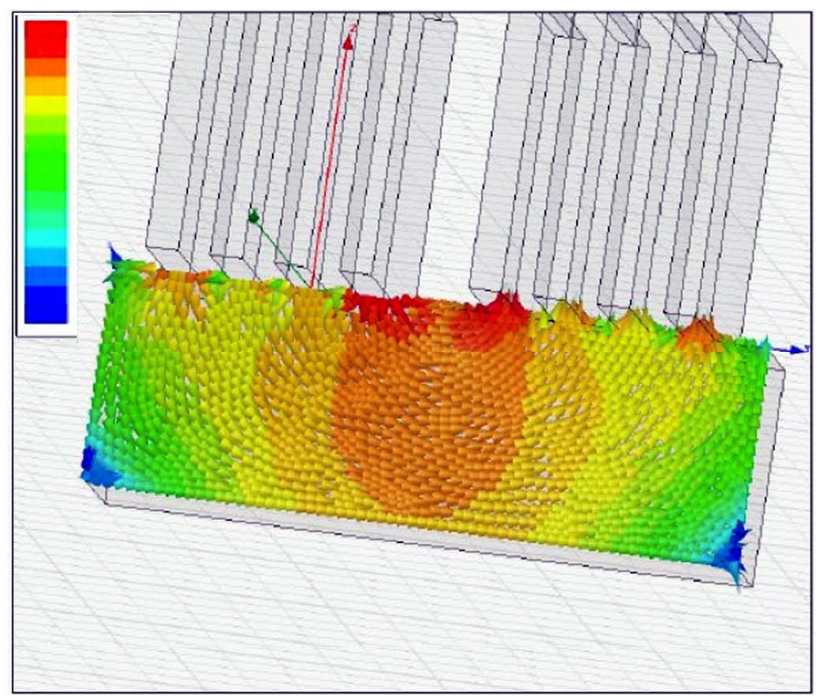

Figure 2. Qualitative electromagnetic field simulation of the tissue effects of simple biolpar RF (left) and mutiple phase controlled RF generators (right). Since the multisource RF handpiece delivers energy in constant circulatory motion the effect will be an average lower temperature on the epidermis $(<43 \mathrm{deg})$ and higher temperature in the lower skin layers, without the need for cooling. This technology allows the system to keep epidermal temperature bellow 43 deg while reaching up to $57 \mathrm{deg}$ in the depth of the tissue. 
Fitzpatrick skin type 3 to 5 were enrolled in this study.

The volunteers did not have a history of any type of skin disease or cosmetic procedure affecting the treatment areas within the last 3 years. Volunteers did not have any specific diet. No volunteers exhibited weight loss during the study periods. No topical pre-treatment medication was used, and the post-treatment skin care regimen consisted of a gentle cleanser and sunblock. All volunteers gave written informed consent for participation in the study after reading the experimental protocol and being advised about the risks of treatments.

\subsection{RF Treatment}

The RF device used in this study was the FDA cleared, EndyMed PRO ${ }^{\mathrm{TM}}$, (Endymed Medical, Cesarea, Israel). This system uses multiple phase-controlled RF generators, emitting RF at $1 \mathrm{MHz}$ frequency, 1 to 65 watts. Twenty Japanese volunteers were treated on both cheeks. Three treatment sessions with a 1-week interval between treatments were performed.

Volunteers were treated at a $33 \mathrm{~W}$ output, which allowed a painless procedure. If the volunteer reported a strong sensation of heat, the handpiece was rotated slightly faster and/or the treatment head was moved slightly away from the point of heat sensation. No topical anesthetics or oral analgesics were administered before, during, or after the treatment. In addition, no skin cooling was required.

\subsection{Objective Assessments}

Digital photographs and 3D imaging with quantitative volume measurements were conducted as objective assessments with a Canfield Scientific Vectra camera and software (Canfield Scientific Inc., Fairfield, New Jersey). This system is designed to accurately capture the surface shape and also 2D color information of the human body. The capture sequence of Vectra was set to less than $3 \mathrm{~ms}$ in order to capture the shape accurately even if the subject was not perfectly still.

A 3D color schematic representation indicates the volume changes between pretreatment and post-treatment images in the face, and shows the varying degrees of tightening achieved in colors ranging from yellow to red. Green areas indicated no changes to the face.

In this study, volume change was observed as volume reduction in the cheek, and recorded in milliliters. Care was taken to ensure similar non-smiling facial tone in both pretreatment and post-treatment photographs.

\subsection{Subjective Volunteer Assessments}

Subjective volunteer assessments were performed using questionnaires in which the volunteers were asked to give their degree of satisfaction in terms of skin laxity and wrinkles based on a 5-point scale ranging from 0 to 4 $(0=$ worse; $1=$ little satisfaction or not satisfied; $2=$ fairly satisfied; 3 = satisfied; and $4=$ very satisfied). Questionnaires were given 3 months after the final treatment.

\subsection{Statistical Analyses}

The differences were examined for statistical significance using the Wilcoxon signed rank test. A $P<0.05$ was set as a cut-off for statistical significance. Data are represented as means \pm standard deviation (SD).

\section{Results}

Objective assessments evaluated by 3D color schematic representation with quantitative volume measurements showed significant improvement after the treatment (Figures 3-5).

The mean volume reduction measured at the last posttreatment visit was $3.878 \pm 2.86 \mathrm{~mL}$. The post-treatment volume significantly reduce compared with pretreatment volume $(P=0.0007)$. Ninety-five percent of volunteers reported satisfaction with the improvement of skin laxity, and ninety percent of volunteers reported satisfaction with the improvement of wrinkles, such as the nasolabial folds (Figure 6).

Subjective volunteer assessments were performed using questionnaires (below). The volunteers were asked to give their degree of satisfaction in terms of skin laxity,
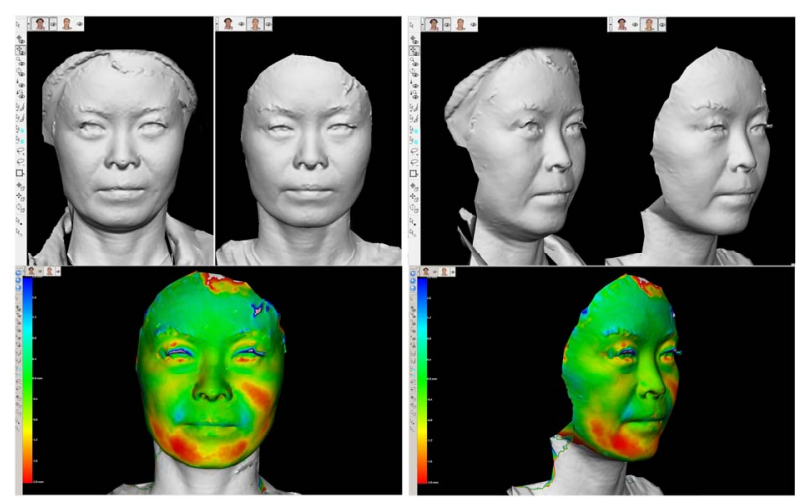

Figure 3. Representative photographs of tightening effects treated with a multisource phase-controlled RF treatment. Pretreatment (Above, left), a 44-year-old Japanese woman exhibited skin laxity in cheek, mental portion, and neck, and wrinkles such as nasolabial fold. Post-treatment (Above, right), significant improvements were noted in both skin laxity and wrinkles. Three-dimensional color schematic representation shows the varying degrees of tightening achieved in colors yellow to red (Below). Green areas remain unchanged. These images indicate significant improvement of appearance, skin laxity, and wrinkles after the treatments. 

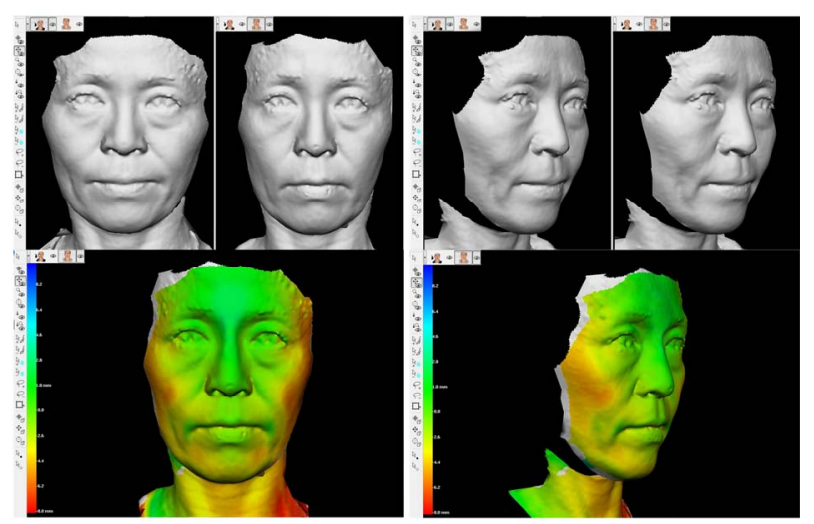

Figure 4. Representative photographs of tightening effects treated with the RF device. Pretreatment (Above, left), a 50year-old Japanese woman exhibited skin laxity in cheek, mental portion, and neck, and nasolabial fold. Post-treatment (Above, right), significant improvements were noted in both skin laxity and wrinkles. Three-dimensional color schematic representation shows significant improvement of appearance, skin laxity, and wrinkles after the treatments.
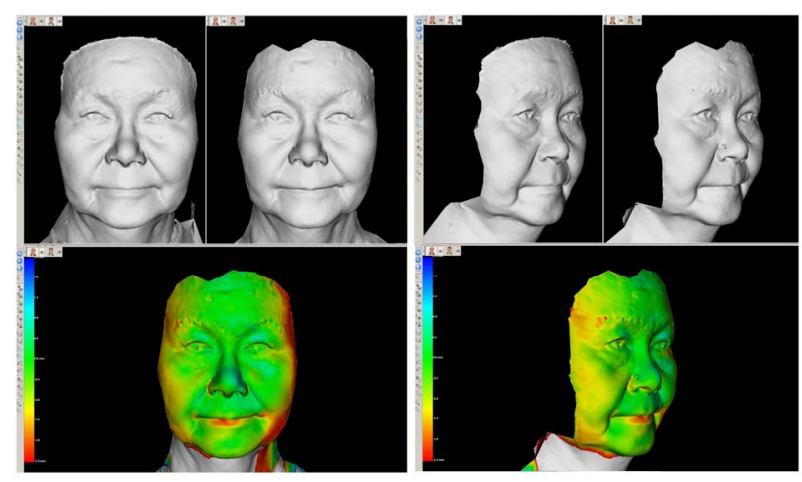

Figure 5. Representative photographs of tightening effects treated with the RF device. Pretreatment (Above, left), a 69year-old Japanese woman exhibited skin laxity in cheek, mental portion, neck, and nasolabial fold. Post-treatment (Above, right), significant improvements were noted in both skin laxity and wrinkles. Three-dimensional color schematic representation shows significant improvement of appearance, skin laxity, and wrinkles after the treatments.

and wrinkles. Subjective volunteer assessments are shown as follows: very satisfied (blue), satisfied (light blue), fairly satisfied (green), and not satisfied (red). Questionnaires were given 3 months after the final treatment. Ninetyfive percent, and $90 \%$ of volunteers were satisfied with the improvements in skin laxity, and wrinkles, respectively.

The mean degrees of satisfaction in terms of skin laxity and wrinkles based on a 5-point scale from 0 to 4 were $3.45 \pm 0.887$ and $3.15 \pm 1.040$, respectively. There was a very good correlation between the volume reduction in the cheeks and patient satisfaction.

Volunteers did not report pain during the RF treatment, even though it was performed without anaesthesia and
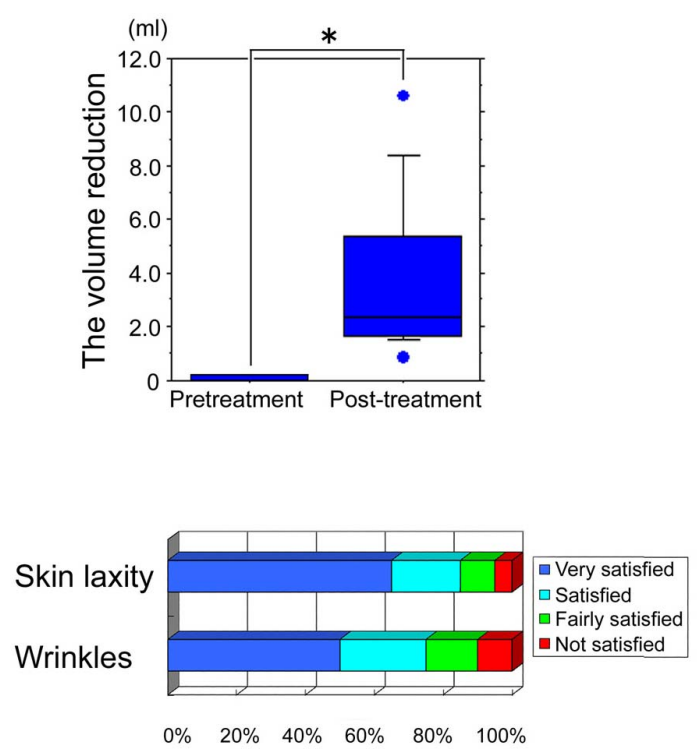

Figure 6. The volume reduction measured by compared the volume at their first pretreatment visit and the last posttreatment visit. The post-treatment volume was significantly reduced compared with pretreatment volume in all the volunteers $(P=0.0007)$ (above). Data represent the means \pm SD. Significant differences are indicated $(*: P<$ 0.05).

contact cooling. Side effects, such as epidermal burns, adipose tissue atrophy, and contraction, were not observed, and the volunteers felt comfortable throughout the study.

\section{Discussion}

Regardless of skin type, skin laxity is one of the most common complaints among aging patients. Although invasive or ablative procedures, such as face-lifts or laser resurfacing, are affective in skin tightening, the downtime and potential adverse effects are not well accepted [18]. Noninvasive skin tightening procedures can be particularly applicable to skin of color, such as Asians, because such procedures are skin-type independent [18]. Furthermore, the aging process of the skin of Asians differs from that of Caucasians, with mid-face aging, such as sagging of the malar fat pads, being a common manifestation [19]. Therefore, skin tightening is an important aspect in the management of skin aging in patients of color [18].

Whereas nonablative intense pulse light skin rejuvenation heats up the superficial dermis, deep tissue heating that involves radiofrequency or near-infrared sources aims to induce thermal injury in the deep tissue [20].

The aim of deep tissue heating is to stimulate new collagen formation, which can achieve skin tightening. Due to its better tissue penetration, RF treatment is capable of volumetric heating of the mid to deep dermis as well as selective heating of the fibrous septa strands and fascia 
layer [21]. Specifically, RF energy heats hydrodermal collagen, promoting both collagen remodeling and skin tightening [22]. Clinically, these effects promote dermal and hypodermal collagen production and the tightening of these deep subcutaneous structures [15,21]. RF devices have been used in thermal delivery systems to provide the beneficial effects of heat while avoiding some of the downfalls of more standard lasers [23].

$\mathrm{RF}$ has been shown to penetrate deeper than optical light sources independent of skin color, and is beneficial for skin tightening. We previously described the tightening effects of near-infrared objectively and histologically, and reported that near-infrared can penetrate deep into human tissue to achieve skin tightening [2-4] and muscle thinning $[5,6]$, as well as non-thermally induce various responses in the skin and subcutaneous tissues [7-12].

In this study, we evaluated the efficacy of multisource phase-controlled RF treatment both subjectively and objectively.

One of the major issues in all skin tightening clinical studies is the lack of an accepted standard regarding the accurate assessment of the degree of skin tightening [18]. Many studies have suggested efficacy of various types of aesthetic devices, but these studies have not included a sufficient objective evaluation. Conventional evaluations using photographs have been widely used, but do not provide accurate objective assessment. In this study, a $3 \mathrm{D}$ photographic system was used to evaluate the amount of post-treatment volume change quantitatively. A 3D imaging approach with quantitative volume measurements (Canfield Scientific Vectra camera and software system) is an effective visual communication tool for skin tightening. Standardized lighting and optically guided object positioning are essential for appropriate pre- and post-treatment evaluation. Photographs are taken from two directions and a $3 \mathrm{D}$ quantitative analysis is systemically performed. Thus, 3D quantitative analysis can evaluate and present the effectiveness and duration of the results objectively as well as show patients results that are not demonstrable with standard, 2D photography.

Although the volume measurement was performed three months after the final treatment, the post-treatment volume was significantly reduced compared to the pretreatment volume in all of the volunteers. Since the effects of this RF treatment are clinically observed for at least several months after the treatment, further studies of volume measurements with a longer follow-up time are needed. Most of the volunteers were satisfied with the improvements in skin laxity and wrinkles, even though the results of the volume measurements were not significant in some volunteers.

Monopolar RF is the first nonablative RF technology shown to be effective for skin tightening $[24,25]$. The original monopolar RF protocols, using single-pass, high fluence regimen, were associated with a greater degree of discomfort and complications, including tissue irregularity and burns [24]. A multipass, low fluence regimen has been developed with the aim of reducing the discomfort of patients and the risk of adverse effects. Avoiding inadvertent overlap of volumetric areas and the use of lower energies with multiple passes may improve efficacy and minimize the risk of unintended thermal injury [26]. However, the low predictability of monopolar RF treatments and-the discomfort that is associated with this procedure is still substantial despite the use of low fluence and multiple passes. Finally, because the device has a disposable tip that can only be used for a single treatment session, the cost effectiveness of this procedure is another important concern.

In bipolar RF one generator is connected to two electrodes on the surface of the skin. In this case the energy delivery will be usually superficial following the shortest way between the electrodes. Other systems with single source of RF connected to 3 or more electrodes (tripolar, multipolar) will provide results similar to bipolar. Both first generation monopolar and bipolar systems are associated with low predictability of treatment results possibly due to large variation of skin impedance causing large variation in the energy delivered into the skin layers.

In this study, a novel multisource phase-controlled system was used, which allows for enhanced energy penetration to lower dermis and hypodermis minimizing epidermal heating. To enhance predictability of energy delivery the system employs a continuous real-time measurement of skin impedance allowing delivery of constant energy to the patient skin independent of changes in its impedance. A $33 \mathrm{~W}$ output was used. Treating each $10 \mathrm{~cm} \times 10 \mathrm{~cm}$ skin area for 30 seconds allows a painless procedure without the need for cooling or anaesthesia. A significant improvement was observed after three rounds of treatment at this output. Each round of treatment consists of six passes. However, more rounds of treatments or a higher output may enhance the effects. Only a few volunteers were unsatisfied with their individual results, and were mainly patients with thicker skin. Therefore, additional rounds of treatment or a higher output may be needed for these patients.

Side effects, such as epidermal burns, adipose tissue atrophy, and contraction, were not observed, and the volunteers felt comfortable throughout the study. Further studies are necessary to determine if a higher output, increased frequency of treatments, or longer periods of treatment may be even more effective in skin tightening.

It should be noted that this was a preliminary study based on a fairly small number of volunteers. We cannot exclude the possibility that intrinstic and extrinstic factors in everyday life may affect the changes demon- 
strated in this study. Therefore further studies in this area are warranted in a larger numbers of patients and with longer post-treatment periods to evaluate variations in treatment parameters and correlations with patients' environmental factors.

\section{Conclusions}

This study examined of first time in Asian population the volumetric changes and subjective effects after treatment with multisource radiofrequency. We found significant improvements in skin laxity through 3 dimensional objective assessments of facial volume. The statistically significant volume reduction measured in treated areas, 3 months after the end of treatment sessions proves a longlasting skin tightening effect probably to collagen remodeling. Efficacy of the treatment was associated with high predictability of the results with remarkably high subjective patient satisfaction rate.

In our view, this study highlights the advantage multisource RF treatment for higher efficacy, reduced discomfort as well as low cost non invasive skin tightening. Our data show that phase-controlled, multisource RF irradiation provides safe and effective treatment of facial skin laxity and for wrinkle reduction in Asian patients.

\section{Acknowledgements}

I thank Dr. Yoram Harth (O. R. Medical Center) for precise data and the file for Figures 1 and 2; Mr. Yohei Nagaoka for three-dimensional imaging performed by a Canfield Scientific Vectra camera and software; and the members of J. Hewitt for helpful comments about EndyMed PROTM, a phase-controlled, multisource RF system.

\section{REFERENCES}

[1] M. Elman and Y. Harth, "Novel Multi-Source PhaseControlled Radiofrequency Technology for Non-Ablative and Micro-Ablative Treatment of Wrinkles, Lax Skin and Acne Scars," Laser Therapy, Vol. 20, No. 2, 2011, pp. 139144. doi:10.5978/islsm.20.139

[2] Y. Tanaka, K. Matsuo, S. Yuzuriha and H. Shinohara, "Differential Long-Term Stimulation of Type I versus Type III Collagen after Infrared Irradiation," Dermatologic Surgery, Vol. 35, No. 7, 2009, pp. 1099-1104. doi:10.1111/j.1524-4725.2009.01194.X

[3] Y. Tanaka, K. Matsuo and S. Yuzuriha, "Long-Term Evaluation of Collagen and Elastin Following Infrared (1100 to $1800 \mathrm{~nm}$ ) Irradiation," Journal of Drugs in Dermatology, Vol. 8, No. 8, 2009, pp. 708-712.

[4] Y. Tanaka, K. Matsuo and S. Yuzuriha, "Long-Term Histological Comparison between Near-Infrared Irradiated Skin and Scar Tissues," Clinical, Cosmetic and Investigational Dermatology, Vol. 3, 2010, pp. 143-149. http://www.dovepress.com/articles.php?article_id=5752

[5] Y. Tanaka, K. Matsuo and S. Yuzuriha, "Long-Lasting
Muscle Thinning Induced by Infrared Irradiation Specialized with Wavelength and Contact Cooling: A Preliminary Report," ePlasty, Vol. 10, 2010, p. e40. $\mathrm{http}: / / \mathrm{www}$. eplasty.com/index.php?option=com content\& view $=$ article $\& i d=453 \&$ catid=171: volume-10-eplasty-2010

[6] Y. Tanaka, K. Matsuo and S. Yuzuriha, "Long-Lasting Relaxation of Corrugator Supercilii Muscle Contraction Induced by Near Infrared Irradiation," ePlasty, Vol. 11, 2011, p. e6.

http://www.eplasty.com/index.php?option=com_content\& view $=$ article \&id=519\&catid=172: volume-11-eplasty-2011

[7] Y. Tanaka, K. Matsuo and S. Yuzuriha, "Objective Assessment of Skin Rejuvenation Using Near-Infrared 1064nm Neodymium: YAG Laser in Asians," Clinical, Cosmetic and Investigational Dermatology, Vol. 4, 2011, pp. 123-130.

http://www.dovepress.com/articles.php?article_id=7972

[8] Y. Tanaka, K. Matsuo and S. Yuzuriha, "Near-Infrared Irradiation Non-Thermally Induces Long-Lasting Vasodilation by Causing Apoptosis of Vascular Smooth Muscle Cells," ePlasty, Vol. 11, 2011, p. e22.

http://www.eplasty.com/index.php?option=com_content \& view=article \&id=541\&catid=172:volume-11-eplasty-2011

[9] Y. Tanaka, K. Matsuo and S. Yuzuriha, "Near-Infrared Irradiation Non-Thermally Affects Subcutaneous Adipocytes and Bones," ePlasty, Vol. 11, 2011, p. e12.

$\mathrm{http}: / /$ www.eplasty.com/index.php?option=com_content\& view=article \&id=528\&catid=172:volume-11-eplasty-2011

[10] Y. Tanaka, K. Matsuo, S. Yuzuriha, H. Yan and J. Nakayama, "Non-Thermal Cytocidal Effect of Infrared Irradiation on Cultured Cancer Cells Using Specialized Device," Cancer Science, Vol. 101, No. 6, 2010, pp. 1396-1402. doi:10.1111/j.1349-7006.2010.01548.x

[11] Y. Tanaka, N. Tatewaki, H. Nishida, T. Eitsuka, N. Ikekawa and J. Nakayama, "Non-Thermal DNA Damage of Cancer Cells Using Near-Infrared Irradiation," Cancer Science, Vol. 103, No. 8, 2012, pp. 1467-1473. doi:10.1111/j.1349-7006.2012.02310.x

[12] Y. Tanaka and K. Matsuo, "Non-Thermal Effects of NearInfrared Irradiation on Melanoma," In: Y. Tanaka, Ed., Breakthroughs in Melanoma Research, InTech, Croatia, 2011, pp. 597-628.

http://www.intechopen.com/books/breakthroughs-in-mela noma-research/non-thermal-effects-of-near-infrared-irradi ation-on-melanoma

[13] N. Sadick and L. Sorhaindo, "The Radiofrequency Frontier: A Review of Radiofrequency and Combined Radiofrequency Pulsed Light Technology in Aesthetic Medicine," Facial Plastic Surgery, Vol. 21, No. 2, 2005, pp. 131-138. doi:10.1055/s-2005-872414

[14] Y. Harth and D. Lischinsky, "A Novel Method for RealTime Skin Impedance Measurement during Radiofrequency Skin Tightening Treatments," Journal of Cosmetic Dermatology, Vol. 10, No. 1, 2011, pp. 24-29. doi:10.1111/j.1473-2165.2010.00535.x

[15] J. Royo de la Torre, J. Moreno-Moraga, A. Munoz and P. C. Navarro, "Multisource, Phase-Controlled Radiofrequency for Treatment of Skin Laxity: Correlation Between Clinical and In-Vivo Confocal Microscopy Results and Real- 
Time Thermal Changes," The Journal of Clinical and Aesthetic Dermatology, Vol. 4, No. 1, 2011, pp. 28-35.

[16] M. Elman, I. Vider, Y. Harth, V. Gottfried and A. Shemer, "Non Invasive Therapy of Wrinkles, Lax Skin Using a Novel Multisource Phase Controlled Radiofrequency System," Journal of Cosmetic and Laser Therapy, Vol. 12, No. 2, 2010, pp. 81-86. doi:10.3109/14764171003706133

[17] N. S. Sadick, M. Sato, D. Palmisano, I. Frank, H. Cohen and Y. Harth, "In Vivo Animal Histology and Clinical Evaluation of Multisource Fractional Radiofrequency Skin Resurfacing (FSR) Applicator," Journal of Cosmetic and Laser Therapy, Vol. 13, No. 5, 2011, pp. 204-209. doi:10.3109/14764172.2011.606467

[18] H. H. Chan, C. S. Yu, S. Shek, C. K. Yeung, T. Kono and W. I. Wei, "A Prospective, Split Face, Single-Blinded Study Looking at the Use of an Infrared Device with Contact Cooling in the Treatment of Skin Laxity in Asians," Lasers in Surgery and Medicine, Vol. 40, No. 2, 2008, pp. 146-152. doi:10.1002/1sm.20586

[19] W. E. Matory, "Skin Care," In: W. E. Matory, Ed., Ethnic Considerations in Facial Aesthetic Surgery, LippincottRaven, Philadelphia, 1998, p. 100.

[20] C. C. Dierickx, "The Role of Deep Heating for Noninvasive Skin Rejuvenation," Lasers in Surgery and Medicine, Vol. 38, No. 9, 2006, pp. 799-807. doi: $10.1002 / 1 \mathrm{sm} .20446$

[21] D. Kist, A. J. Burns, R. Sanner, J. Counters and B. Zelickson, "Ultrastructural Evaluation of Multiple Pass Low Energy versus Single Pass High Energy Radio-Frequency Treatment," Lasers in Surgery and Medicine, Vol. 38, No.

\section{2, 2006, pp. 150-154. doi:10.1002/1sm.20303}

[22] B. D. Zelickson, D. Kist, E. Bernstein, D. B. Brown, S. Ksenzenko, J. Burns, S. Kilmer, D. Mehregan and K. Pope, "Histological and Ultrastructural Evaluation of the Effects of a Radiofrequency Based Nonablative Dermal Remodeling Device: A Pilot Study," Archives of Dermatology, Vol. 140, No. 2, 2004, pp. 204-209. doi:10.1001/archderm.140.2.204

[23] B. D. Owens, B. J. Stickles and B. D. Busconi, "Radiofrequency Energy: Applications and Basic Science," The American Journal of Orthopedics, Vol. 32, No. 3, 2003, pp. 117-120.

[24] R. Fitzpatrick, R. Geronemus, D. Goldberg, M. Kaminer, S. Kilmer and J. Ruiz-Esparza, "Multicenter Study of Noninvasive Radiofrequency for Periorbital Tissue Tightening," Lasers in Surgery and Medicine, Vol. 33, No. 4, 2003, pp. 232-242. doi:10.1002/1sm.10225

[25] M. A. Bogle, N. Ubelhoer, R. A. Weiss, F. Mayoral and M. S. Kaminer, "Evaluation of the Multiple Pass, Low Fluence Algorithm for Radiofrequency Tightening of the Lower Face," Lasers in Surgery and Medicine, Vol. 39, No. 3, 2007, pp. 210-217. doi:10.1002/lsm.20472

[26] A. Willey, R. R. Anderson, J. L. Azpiazu, A. D. Bakus, R. J. Barlow, J. S. Dover, J. M. Garden, S. L. Kilmer, N. Landa, D. Manstein, E. V. Ross Jr., N. Sadick, E. A. Tanghetti, D. Yaghmai and B. D. Zelickson, "Complications of Laser Dermatologic Surgery," Lasers in Surgery and Medicine, Vol. 38, No. 1, 2006, pp. 1-15. doi:10.1002/1sm.20286 